\title{
Research on Anti-Rollover Control for Vehicles under Limit Conditions
}

\author{
Yanfei Li ${ }^{1, a}$, En-guo Dong ${ }^{2, \mathrm{~b}}$ and Lei Zhang ${ }^{3, \mathrm{c}}$ \\ ${ }^{1}$ College of Automobiles and Transportation, \\ Tianjin University of Technology and Education, Tianjin, China

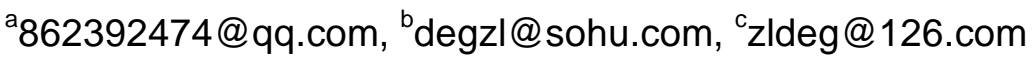

Keywords: Vehicle rollover; limit conditions; yaw rate; simulation

Abstract. To improve the anti-rollover capability of vehicles under limit conditions, the rollover stability of vehicles in high speed steering was studied; a vehicle dynamics model was built with the software of Carsim. Based on the variable threshold method of yaw rate, an anti-rollover controller was designed, and the yaw moment was modified to regulate wheel braking force in order to achieve the excellent active anti-rollover. The model of the vehicle was stability controlled in the software of Simulink. Through the comparison of simulation test, the controller can improve the anti-rollover ability of vehicles under limit conditions, and improve the vehicle handling stability.

\section{Introduction}

Rollover is an extremely dangerous lateral movement of vehicles in the process of driving, due to the centrifugal force and the loss of driving stability. Research shows that under limit conditions such as sharp turns in high speed or low adhesion road, it is prone to rollover for SUV and heavy semi-trailers, whose centroid is high, track is narrow ${ }^{[1]}$. The anti-rollover control of the vehicle has become a focus of research in the field of vehicle active safety.

In recent years, in order to reduce the loss caused by rollover accident and further enhance the active safety of vehicles, domestic and foreign scholars and automobile manufacturers have carried out a lot of work on vehicle rollover control problems. Some methods for improving the vehicle rollover stability are put forward, such as differential braking control ${ }^{[2]}$ and semi-active suspension control ${ }^{[3]}$. But the anti-rollover effect of these researches are not ideal, especially under limit conditions such as sharp turning with high speed and low adhesion road, and most of the studies are still in the stage of theoretical research. In this article, the vehicle model is built by the software of Carsim, and an anti-rollover controller is designed based on the variable threshold method of yaw rate with the software of Simulink. Finally, the combined simulation of vehicle model and anti-rollover controller is carried out under fishhook condition to verify the validity of the control strategy.

\section{Vehicle model}

Carsim is a parametric modeling and simulation software developed by Mechanical Simulation Corporation. Carsim vehicle model includes seven subsystems: body, aerodynamics, transmission assembly, brake system, steering system, tire and suspension. Vehicle model is established without definition of each component of a specific structural form, and users only need to select the component module to define the related parameters that can reflect the performance of the components ${ }^{[4]}$. The interface of body parameters modeling is shown in Figure1. 


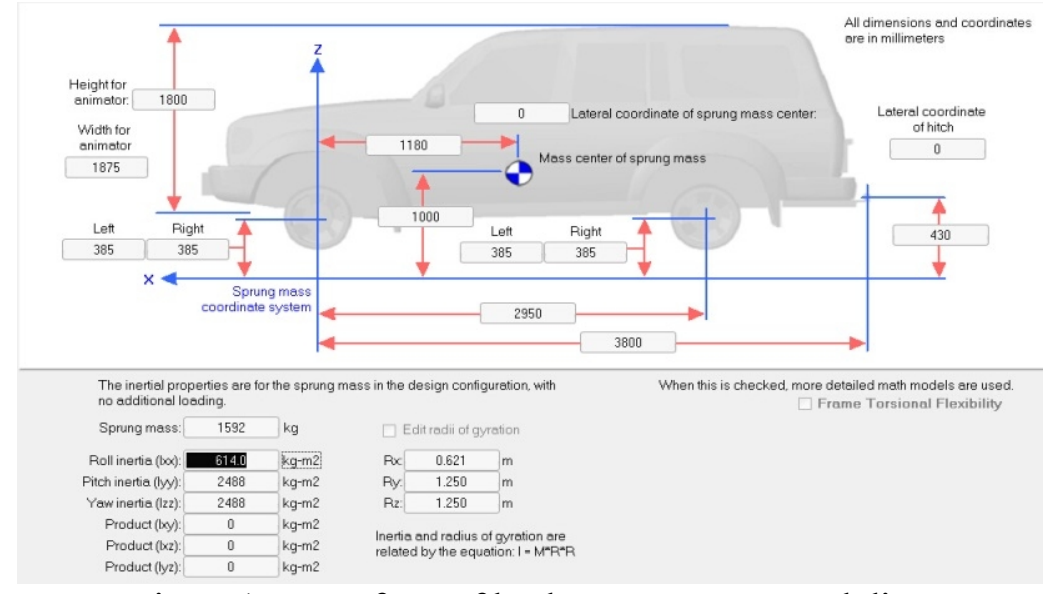

Figure1. Interface of body parameters modeling

The simulation selects a high centroid SUV as the experimental object, then set the cylinder pressure of four brake wheel (left front, right front, left and right after) as Carsim input variables, and output variables are wheel angle, vehicle speed and yaw rate. The main parameters of the vehicle are shown in Table 1.

Table1. Main parameters of vehicle

\begin{tabular}{cc}
\hline Parameters & Configuration \\
\hline vehicle outlook: length / width / height $/ \mathrm{mm}$ & $3800 / 1875 / 1800$ \\
wheelbase/mm & 2950 \\
minimum ground distance $/ \mathrm{mm}$ & 250 \\
curb mass $/ \mathrm{kg}$ & 1592 \\
front/rear tread/mm & $1575 / 1575$ \\
tire specifications & $255 / 75 \mathrm{R} 16$ \\
braking system & four wheel braking + ABS \\
steering system & rack and pinion steering gear \\
transmission system & four-wheel drive \\
suspension system & independent suspension \\
spring stiffness $/(\mathrm{N} / \mathrm{mm})$ & 146 \\
shock absorber & Big SUV Damping \\
\hline
\end{tabular}

\section{Rollover avoidance control}

Anti-rollover controller set yaw rate as control variable, and it is adjusted to proper range through comparing the actual output parameters and the ideal parameters, then control the side force to meet the requirements of the vehicle stability. In order to obtain the ideal yaw rate, a vehicle model with linear two degree of freedom is established, as shown in Figure 2. Neglect the effect of the steering system, directly set front wheel angle as input; ignore the role of suspension, the vehicle just do plane motion parallel to the ground; the vehicle forward speed along the $\mathrm{X}$ axis $(\mathrm{u})$ is regarded as invariant ${ }^{[5]}$.

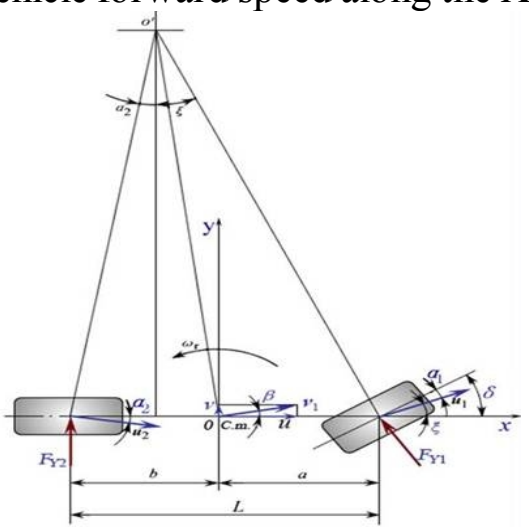

Figue2. 2-DOF vehicle model

According to the two degree of freedom vehicle model, motion differential equation can be 
established:

$$
\begin{aligned}
& \left(k_{1}+k_{2}\right) \beta+\frac{1}{u}\left(a k_{1}-a k_{2}\right) \omega_{r}-k_{1} \delta=m\left(\dot{v}+u \omega_{r}\right) \\
& \left(a k_{1}-b k_{2}\right) \beta+\frac{1}{u}\left(a^{2} k_{1}+b^{2} k_{2}\right) \omega_{r}-a k_{1} \delta=I_{z} \dot{\omega}_{r}
\end{aligned}
$$

The value of yaw rate $\omega_{r}$ is constant when the vehicle is in steady-state, at this point, $\dot{v}=0, \dot{\omega}_{r}=0$, and put them into formula (1) and (2), the nominal yaw rate $\omega_{r}$ can be calculated:

$$
\omega_{r}=\frac{u / L}{1+\frac{m}{L^{2}}\left(\frac{a}{k_{2}}-\frac{b}{k_{1}}\right) u^{2}} \delta=\frac{u / L}{1+K u^{2}} \delta
$$

In the formula (3), $K=\frac{m}{L^{2}}\left(\frac{a}{k_{2}}-\frac{b}{k_{1}}\right)$, known as the stability factor, $\mathrm{u}$ is vehicle speed and $\mathrm{L}$ is the wheelbase, $\delta$ denotes the front wheel angle.

When the values of actual yaw rate $\omega_{n}$ and nominal yaw rate $\omega_{r}$ are equal, vehicle shows good control stability, with slight understeer; when $\omega_{n}$ is small, the vehicle is in steady-state, the controller does not need to work at this time; therefore, set an initial value $\omega_{0}$ for the controller, only when $\left|\omega_{n}\right|>\omega_{0}$, the controller only start control.

$$
a_{y}=\dot{v}+u \omega
$$

In the formula (4), $a_{y}$ is the centroid lateral acceleration, and $\dot{v}$ denotes the change rate of lateral acceleration. When vehicle rollover doesn't occur, $v$ is very small compared with $u \omega$, the limit value of yaw rate $\omega_{\max }=\varepsilon a_{y \max } / u, a_{y \text { max }}$ denotes the theory maximum value of vehicle centroid acceleration, and $\varepsilon$ is a coefficient considering the influence of $\dot{v}$, desirable $\varepsilon=0.85^{[6]}$. Only when $\left|\omega_{n}\right|<\left|\omega_{\max }\right|$, the vehicle can drive in a stable state, the target of yaw moment control is to make $\omega_{n}$ in the vicinity of $\omega_{r}$ under the premise of vehicle stability driving.

In conclusion, when the anti-rollover controller enters the working state, the ideal yaw rate can be obtained:

$$
\omega_{\text {des }}= \begin{cases}\omega_{r}, & \left|\omega_{r}\right|<\left|\omega_{\max }\right| \\ \omega_{\max } \operatorname{sign}\left(\omega_{r}\right), & \left|\omega_{r}\right| \geq\left|\omega_{\max }\right|\end{cases}
$$

In the formula (5), $\omega_{\text {des }}$ denotes the ideal yaw rate and $\operatorname{sign}\left(\omega_{r}\right)$ is a function of $\omega_{r}$.

To compare with the actual yaw rate $\omega_{\mathrm{n}}$ and the nominal yaw rate $\operatorname{sign}\left(\omega_{r}\right)$, find out their difference $\Delta \omega, \Delta \omega=\left(\omega_{\mathrm{n}}-\omega_{\text {des }}\right) \operatorname{sign}\left(\omega_{\text {des }}\right)$, in the formula, $\operatorname{sign}\left(\omega_{\text {des }}\right)$ is a function of $\omega_{\text {des }}$. By comparing the obtained $\Delta \omega$ to the preset values of positive threshold $\Delta \omega^{+}$or negative threshold $\Delta \omega^{-}$, then judge the direction of the vehicle turn combined with the positive and negative of $\delta$ (when $\delta$ is positive, the vehicle turn left, $\delta$ is negative, the vehicle turn right). Finally, control the wheel cylinder pressure of the steering wheel to prevent vehicle rollover. The control mode of different vehicle driving state is shown in Table 2.

Table2. Control strategy

\begin{tabular}{c|c}
\hline Vehicle driving state & Control strategy \\
\hline$\Delta \omega>\Delta \omega^{+}, \delta>0$ (oversteer) & Increase the braking force of the right front wheel \\
\hline$\Delta \omega<\Delta \omega^{-}, \delta>0$ (understeer) & Increase the braking force of the left rear wheel \\
\hline$\Delta \omega>\Delta \omega^{+}, \delta<0$ (oversteer) & Increase the braking force of the left front wheel \\
\hline$\Delta \omega<\Delta \omega^{-}, \delta<0$ (understeer) & Increase the braking force of the right rear wheel \\
\hline$\Delta \omega^{-}<\Delta \omega<\Delta \omega^{+}$ & No control \\
\hline
\end{tabular}




\section{Simulation results}

In order to verify the validity and practicality of the controller, the combined simulation of anti-rollover controller and Carsim vehicle model was created, as shown in Figure 3, then the vehicle with anti-rollover controller and the vehicle without controller were compared. Two E-Class, CG SUV: High models in the software of Carsim were selected for simulation.

The initial conditions of simulation are: the speed of $100 \mathrm{~km} / \mathrm{h}$, the adhesion coefficient of the pavement is 0.85 , no braking, the shifting control is closed loop shift control at 4th model, and the steering wheel angle range is $-294^{\circ}-294^{\circ}$, open-loop control model.

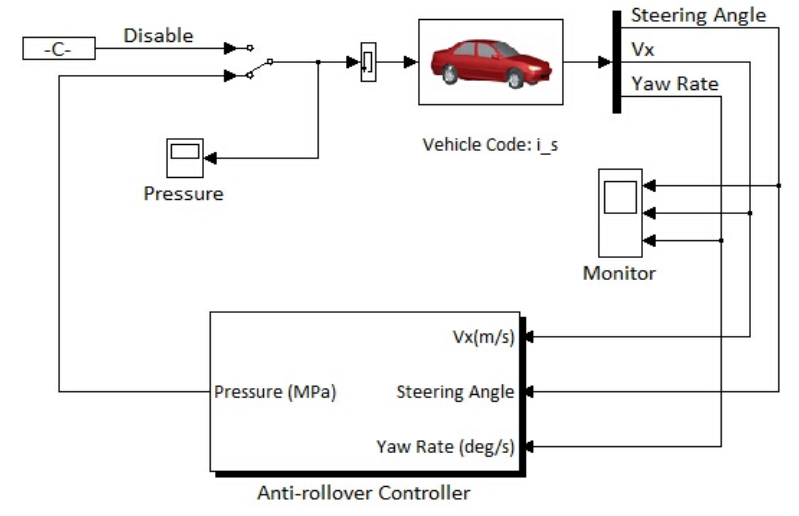

Figure3. Combined simulation model

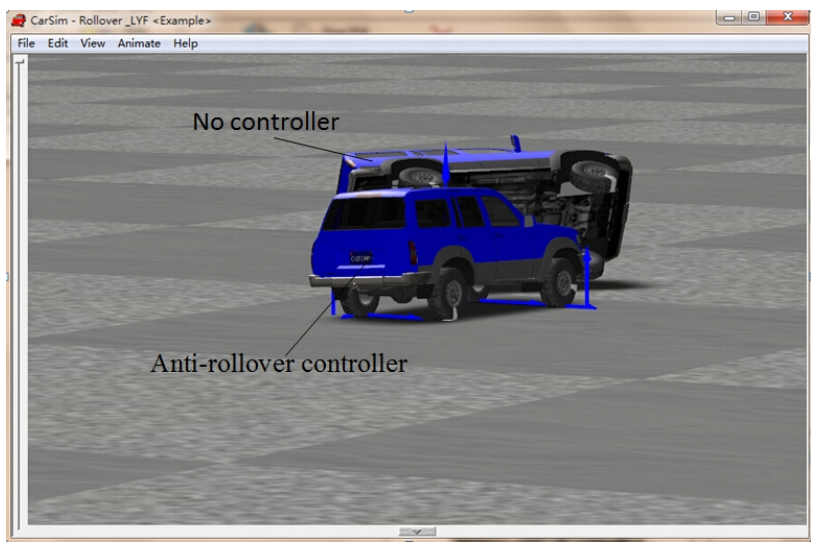

Figure4. Vehicle running state

Control the anti-rollover controller in Simulink, mainly to complete the simulation of vehicle driving under limit conditions, to prevent vehicle rollover and improve vehicle handling stability. The test parameters include roll angle, roll rate and lateral acceleration.

Vehicle running state as shown in Figure4, the rollover occurred on the vehicle without controller, and the vehicle applied with anti-rollover controller without the occurrence of rollover. Specific simulation parameters are shown in Figure5 Figure7.

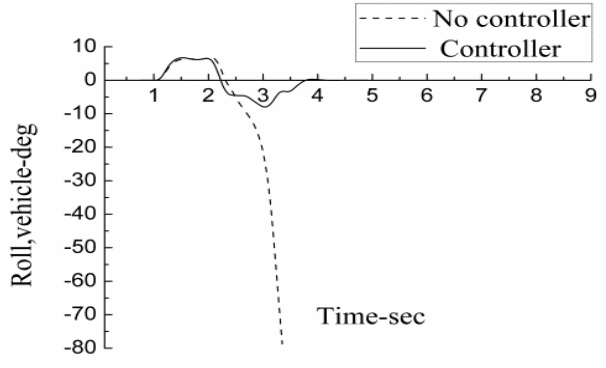

Figure5. Vehicle roll angle

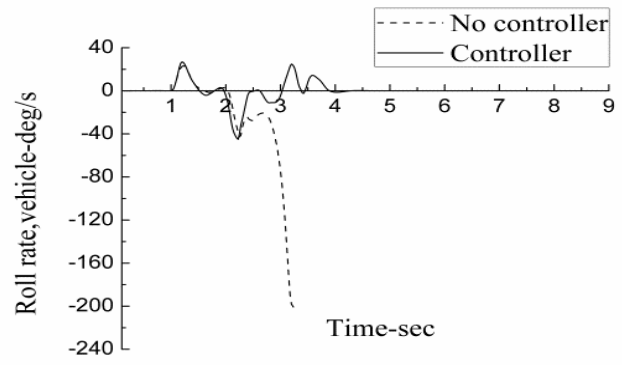

Figure6. Vehicle roll rate

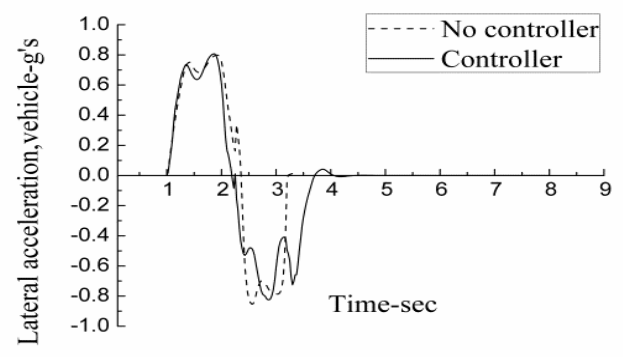

Figure7. Vehicle lateral acceleration

In Figure5, the vehicle without controller rollover happened in 3 seconds; the vehicle applied with anti-rollover controller, its biggest roll angle is $8^{\circ}$, vehicle body roll slightly at this time, but it will be reduced to 0 soon, improve the lateral stability of vehicle. Figure6 shows that roll rate under the control 
of anti-rollover controller reduced to $40 \% \mathrm{~s}$, the controller prevent the car rollover, and reduce the speed of the vehicle roll. The lateral acceleration under the anti-rollover controller also has a certain degree of lower and running state back to normal in 4 seconds in Figure7. By contrast, the anti-rollover controller significantly reduces the roll angle, roll rate and lateral acceleration, greatly improving the lateral stability and ride performance of the vehicle, and reduce the risk of rolling over.

\section{Conclusions}

Anti-rollover controller was designed, which took yaw rate as the control target; through the combined simulation of Carsim vehicle model and the controller, the controller's ability to prevent vehicles rollover was verified. The controller was built based on the variable threshold method of yaw rate and differential braking principle; by comparison with the vehicle without controller under fishhook condition, the simulation verified the validity of the control strategy. Under the control of anti-rollover controller, the large amplitude of vehicle roll angle, roll rate, lateral acceleration and other parameters were improved to a great extent, and the controller improved the vehicle handling stability and ride performance.

\section{Acknowledgements}

Supported by National Natural Science Foundation of China (Grant No. 51005167), Tianjin University of Technology and Education advanced project (Grant No. KJY14-01).

\section{References}

[1] Litong Guo. Research on active anti-rollover systems based on integrated chassis control for SUV [D].Changchun: Jilin University, 2013. (In Chinese)

[2] Chen B C, Peng H. Differential-braking-based Rollover Prevention for Sport Utility Vehicles with Human-in-the-loop Evaluations [J]. Vehicle System Dynamics, 2001, 36(4-5): 359-389.

[3] Guglie lmino E, Sireteanu T, Stammers C W, et al. Semi-active suspensions control: improved vehicle ride and road friendliness [M]. Springer London, 2008.

[4] Limin Niu, Jichan $\mathrm{Xu}$, Chao Liu. Research on simulation model for suspension and steering system based on Carsim [J]. Mechanical Science and Technology for Aerospace Engineering, 2014, 33(4): 573-577. (In Chinese)

[5] Zhisheng Yu. Automobile theory [M]. Beijing: China Machine Press, 2000. (In Chinese)

[6] Rajamani, Rajesh Vehicle Dynamics and Control [M].Springer, 2006. 